\title{
EFECTO DE LA INMUNOCASTRACIÓN EN EL PESO CANAL Y LAS PIEZAS NOBLES EN CERDAS IBÉRICAS DE CEBO
}

\author{
IMMUNOCASTRATION EFFECT ON THE WEIGHT OF CARCASS AND MAIN LEAN CUTS \\ IN IBERIAN FEMALES
}

\author{
Gómez-Fernández, J. ${ }^{1 *}$; Tomás, C. ${ }^{2}$; Gómez-Izquierdo, E. ${ }^{1}$ y Mercado, E. de ${ }^{1}$
}

${ }^{1}$ Centro de Pruebas de Porcino. Instituto Tecnológico Agrario. Consejería de Agricultura y Ganadería. Junta de Castilla y León. Hontalbilla, Segovia. España. *gomferjs@itacyl.es

${ }^{2}$ CITA-IVIA. Castellón. España.

Palabras clave adicionales

Jamón. Lomo. Paleta. Rendimiento.

\section{RESUMEN}

Se utilizaron 192 hembras Ibéricas (cruzadas, Duroc*lbérica), con 110 días de edad y $45 \pm 3,41$ $\mathrm{kg}$ de peso vivo para estudiar el efecto de la inmunocastración en el rendimiento de la canal y de las piezas nobles. Los animales experimentales se distribuyeron en 3 tratamientos: hembras castradas quirúrgicamente (CC), hembras enteras $(\mathrm{CE})$ y hembras inmunocastradas $(\mathrm{Cl})$, siendo el diseño experimental en bloques completos al azar, con 48 réplicas totales de 4 cerdas cada una, 12 réplicas por bloque y 16 por tratamiento. El pienso fue el mismo para todos los animales, suministrándose ad libitum. Una vez realizado el sacrificio de los animales, se seleccionaron 31 hembras por cada tratamiento para evaluar la canal y el rendimiento de las piezas nobles. Las cerdas $\mathrm{Cl}$ tendieron a presentar un mayor peso de sacrificio y peso de canal $(p<0,10)$, siendo los pesos de jamones y paletas similares a las hembras CE $(p>0,05)$, y significativamente menos pesados en el grupo de las hembras CC $(p<0,05)$. Por el contrario, el peso de los lomos resultó significativamente mayor en las hembras CE que en las $\mathrm{Cl}$ y CC $(3,05$ vs. 2,$82 ; 2,71 \mathrm{~kg}$, respectivamente; $p<0,05)$. Se calcularon las ecuaciones de regresión relacionando el peso de la canal con el peso del jamón, la paleta y el lomo para cada tratamiento. Las mejores relaciones entre las variables se obtuvieron con funciones lineales en el caso del jamón y la paleta $\left(R^{2}>55 \%\right.$; $\left.p<0,05\right)$ y, en el caso del lomo, lineal y no significativa en el grupo $C C(p>0,05)$ y cuadráticas para los grupos CE y Cl

\author{
AdDitional KEYWORDS \\ Foreleg. Ham. Loin. Performance.
}

$(p<0,05)$. La inmunocastración favorece el crecimiento de las cerdas y que la castración quirúrgica no presenta ninguna ventaja productiva.

\section{SUMMARY}

A total of 192 lberian females (Duroc* ${ }^{*}$ lberian), with 110 days of age and $45 \pm 3.41 \mathrm{~kg} \mathrm{BW}$, were used to study the effect of immunocastration in yield of carcass and prime lean cuts. There were three treatments: surgically castrated females (CC), entire females (CE), and inmunocastrated females $(\mathrm{Cl})$ the experimental design was a complete randomized blocks with 48 replicates total ( 4 pigs in each), 12 replicates per block and 16 per treatment. The feed, the same for all animals, was suministered ad libitum. After sacrifice, 31 pigs were selected for each treatment to assess the performance of carcass and main lean cuts. Treatment $\mathrm{Cl}$ tended to improve slaughter and carcass weights $(p<0.10)$, with the same weight of hams and forelegs that CE $(p>0.05)$ and significantly less in the $C C(p<0.05)$. By contrast, the loins were heavier in CE than in $\mathrm{Cl}$ and CC $(3.05$ vs. $2.82,2.71 \mathrm{~kg}$, respectively; $p<0.05)$. Regression equations were established relating the carcass weight with hams, forelegs and loins weights. The best relationships between variables were obtained with linear functions in the case of ham and foreleg $\left(R^{2}>55 \% ; p<0.05\right)$. For loin, linear and non-significantly in $C C(p>0.05)$ and quadratic for $\mathrm{CE}$ and $\mathrm{Cl}(\mathrm{p}<0.05)$. The immunocastration favors 


\section{GÓMEZ-FERNÁNDEZ, TOMÁS, GÓMEZ-IZQUIERDOY MERCADO}

the growth of Iberian female; the surgical castration is the least interesting option.

\section{INTRODUCCIÓN}

El RD 1469/2007 establece que las piezas que se destinen a la elaboración de los productos ibéricos procedan de cerdos con una edad de sacrificio mínima entre 10 y 14 meses, en función del sistema de explotación que se haya seguido. El sacrificio a esas edades es posterior a la pubertad y no evita comportamientos indeseables, sobre todo en extensivo, que comprometan la bioseguridad de la granja. Además, puede ocasionar la aparición de olor sexual en los productos cárnicos derivados, como ocurre también en otras razas (EFSA, 2004). Todo ello justificó la práctica general (Aparicio, 1977) de la castración de machos y hembras de raza Ibérica. Al mismo tiempo, el citado $\mathrm{RD}$ admite que los cerdos cuya carne se comercialice con el amparo de la Norma de Calidad puedan resultar del cruce de cerdas Ibéricas con machos Duroc. Actualmente, este tipo de producción, cruzada e intensiva es mayoritaria en el Ibérico (MAGRAMA, 2012), la castración de las cerdas continúa sin que se haya demostrado completamente sus ventajas (Mateos et al., 2009), aunque se ha propuesto (Latorre, 2011) para mejorar la calidad de la canal y de la carne en otras razas.

La castración de los animales es un requisito del mercado para ciertos productos (European Declaration on alternatives to surgical castration of pigs, 2010) aunque, el rechazo social, las consideraciones de bienestar animal, y las propias limitaciones legales (Directiva 120/2008/CE del Consejo) obligan al sector a encontrar procedimientos alternativos, que sustituyan la castración como manejo rutinario.

La inmunocastración ha sido ensayada en múltiples situaciones (Batorek et al., 2012), aunque nunca en cerdas de razas mediterráneas como la Ibérica. Por lo tanto, el objetivo del ensayo, fue valorar el efecto zootécnico del estado reproductivo (castradas quirúrgicamente, enteras, inmunocastradas) de cerdas Ibéricas cruzadas, explotadas en régimen intensivo, sobre el peso de sacrificio, el peso de la canal y su relación con el peso de las piezas nobles.

\section{MATERIAL Y MÉTODOS}

El ensayo tuvo lugar en el Centro de Pruebas de Porcino del Instituto Tecnológico Agrario de Castilla y León (Consejería de Agricultura y Ganadería, Junta de Castilla y León), situado en Hontalbilla, Segovia, cumpliendo las directrices del RD 1201/2005, de 10 de octubre, sobre protección de los animales utilizados para experimentación y otros fines científicos. Se utilizaron 192 cerdas Ibéricas (Duroc x Retinta) con 110 días de vida y 44,94 $\pm 3,41 \mathrm{~kg}$ de peso vivo. Al día siguiente de su llegada, se identificaron y pesaron individualmente, distribuyéndose en función del peso y estado reproductivo, asignándose a los distintos tratamientos experimentales: cerdas castradas quirúrgicamente (CC), cerdas enteras (CE) y cerdas inmunocastradas (CI). Las cerdas $\mathrm{CC}$ se castraron a las 8 semanas de edad y las cerdas CI recibieron (EMA, 2011) dos dosis de Improvac ${ }^{\circledR}$ a los 60 y $84,2 \mathrm{~kg}$ de peso vivo (18 y 22 semanas de edad). El diseño experimental fue en bloques completos al azar, con 48 réplicas totales de 4 cerdas cada una, 12 réplicas por bloque, y 16 por tratamiento. Todos los animales consumieron el mismo pienso (tabla I), suministrado ad libitum; el cambio de pienso de cebo a acabado se hizo a los $94,73 \pm 0,999 \mathrm{~kg}$ de peso vivo. Tras su envío a matadero, se seleccionaron 31 cerdas, coincidentes con el peso medio de cada réplica, con el fin de valorar la canal (entera) y las piezas nobles (promedio de ambos lados). Las canales fueron procesadas de acuerdo al método europeo de referencia (Branscheid et al., 1990). Los datos se analizaron con los procedimientos GLM, REG y CORR del programa SAS Institute (2004), con el siguiente modelo para el análisis de 
Tabla I. Composición (\%) de los piensos. (Composition (\%) of diets).

\begin{tabular}{lccccccc}
\hline & MS & PB & GB & FB & C & Lys & EN \\
\hline Cebo & 89,9 & 14,7 & 7,0 & 5,8 & 5,2 & 0,72 & 2445 \\
Acabado & 89,9 & 11,5 & 8,4 & 5,0 & 6,7 & 0,56 & 2484
\end{tabular}

${ }^{1} \mathrm{MS}=$ materia seca; $\mathrm{PB}=$ proteína bruta; $\mathrm{GB}=$ grasa bruta; $\mathrm{FB}=$ fibra bruta; $\mathrm{C}=$ cenizas; $\mathrm{Lys}=$ lisina; $\mathrm{EN}=$ energía neta $\mathrm{kcal} / \mathrm{kg}$.

varianza de calidad de la canal (la unidad experimental fue el animal):

$$
\mathrm{Y}_{\mathrm{ij}}=\mu+\operatorname{EREPRO}_{\mathrm{i}}+\varepsilon_{\mathrm{ij}}
$$

donde:

$\mathrm{Y}=$ peso canal caliente, rendimiento canal, rendimiento de piezas nobles -peso y porcentaje; $\mu=$ media general;

EREPRO= estado reproductivo (CC, $\mathrm{CE}$ y $\mathrm{Cl})$; $\varepsilon=$ error residual.

Igualmente, se calcularon las ecuaciones de regresión de mejor ajuste.

\section{RESULTADOSYDISCUSIÓN}

Las hembras CI presentaron una tendencia $(p<0,10)$ tanto a un mayor peso de
Tabla II. Pesos (kg) de sacrificio (PS), de canal $(P C)$ y piezas nobles (J: jamón, P: paleta, L: lomo), en cerdas castradas quirúrgicamente $(C C)$, enteras $(C E) e$ inmunocastradas (CI). (Slaughter (PS), carcass (PC) and major cuts (J: Ham, P: Foreleg, L: Loin) weights $(\mathrm{kg})$, in surgically castrated (CC), entires (CE) and inmunocastrated (Cl) sows).

\begin{tabular}{lccccc}
\hline & CC & CE & Cl & EEM & $\mathrm{p}$ \\
\hline PS & 161,33 & 165,21 & 169,96 & 2,63 & $\mathrm{t}$ \\
PC & 127,67 & 129,05 & 133,89 & 2,16 & $\mathrm{t}$ \\
$\mathrm{J}$ & $12,57^{\mathrm{a}}$ & $13,50^{\mathrm{b}}$ & $13,27^{\mathrm{b}}$ & 0,19 & $* \star$ \\
$\mathrm{P}$ & $7,59^{\mathrm{a}}$ & $8,28^{\mathrm{b}}$ & $8,02^{\mathrm{b}}$ & 0,11 & $* * *$ \\
$\mathrm{~L}$ & $2,71^{\mathrm{a}}$ & $3,05^{\mathrm{b}}$ & $2,82^{\mathrm{a}}$ & 0,05 & $* * *$ \\
\hline
\end{tabular}

$\mathrm{t}=\mathrm{p}<0,10 ;{ }^{* *} \mathrm{p}<0,01 ;{ }^{* * *} \mathrm{p}<0,001 . \mathrm{N}=93 ; \mathrm{n}=31$. a,b Letras distintas en la misma fila indican diferencias significativas.

sacrificio (PS) como a un mayor peso de la canal (PC) (tabla II), mientras que los pesos de los jamones y paletas fueron comparables en los tratamientos CE y CI $(p>0,05)$ y significativamente menores en el grupo CC $(\mathrm{p}<0,05)$. Los lomos de las hembras CE pesaron más que en los grupos CC y CI. Las ecuaciones de regresión (tabla III), para pesos de canal con rangos entre 104,4-151,4 $\mathrm{kg}(\mathrm{CC}), 90-156,8 \mathrm{~kg}(\mathrm{CE})$ y $113,8-153 \mathrm{~kg}(\mathrm{CI})$,

Tabla III. Ecuaciones de regresión lineal y cuadrática entre los pesos de la canal $(P C)$ y de las piezas nobles (J: jamón, P: paleta, L: lomo). (Linear and quadratic relations between carcass weight (PC) and weight of major cuts (J: Ham, P: Foreleg, L: Loin)).

\begin{tabular}{|c|c|c|c|c|}
\hline Ecuaciones de regresión & & $\mathrm{R}^{2}$ & $\mathrm{RSD}$ & significación \\
\hline \multirow[t]{3}{*}{ Castradas quirúrgicamente (CC) } & $\mathrm{J}=4,96+0,059 \cdot \mathrm{PC}$ & 0,57 & 0,55 & $* \star \star$ \\
\hline & $P=3,33+0,033 \cdot P C$ & 0,44 & 0,47 & 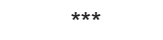 \\
\hline & $\mathrm{L}=1,94+0,006 \cdot \mathrm{PC}$ & 0,06 & 0,25 & NS \\
\hline \multirow[t]{3}{*}{ Enteras (CE) } & $\mathrm{J}=4,77+0,067 \cdot \mathrm{PC}$ & 0,72 & 0,61 & $* * *$ \\
\hline & $P=3,55+0,036 \cdot P C$ & 0,62 & 0,41 & $\star \star \star *$ \\
\hline & $\mathrm{L}=-5,93+0,125 \cdot \mathrm{PC}-0,0004 \cdot \mathrm{PC}^{2}$ & 0,62 & 0,22 & *** \\
\hline \multirow[t]{3}{*}{ Inmunocastradas (Cl) } & $\mathrm{J}=1,75+0,086 \cdot \mathrm{PC}$ & 0,59 & 0,77 & $* \star *$ \\
\hline & $P=2,06+0,044 \cdot P C$ & 0,56 & 0,42 & $\star \star * \star$ \\
\hline & $\mathrm{L}=-9,21+0,16 \cdot \mathrm{PC}-0,0005 \cdot \mathrm{PC}^{2}$ & 0,37 & 0,29 & ** \\
\hline
\end{tabular}

$R^{2}=$ coeficiente de determinación; $R S D=$ desviación residual estándar. $N S p>0,10 ;{ }^{* *} p<0,01$; ${ }^{* *} p<0,001$. $\mathrm{N}=93 ; n=31$. 


\section{GÓMEZ-FERNÁNDEZ, TOMÁS, GÓMEZ-IZQUIERDOY MERCADO}

muestran los mejores ajustes con funciones lineales y cuadráticas. La variabilidad explicada por PC fue, en general, aceptable (>44\%), si bien los máximos valores correspondieron al grupo $\mathrm{CE}$, para todas las piezas, lo que no deja de ser llamativo, ya que son cerdas que presentan varios celos antes del sacrificio y se supone que son más heterogéneas. La predicción del lomo en el grupo CC no fue significativa, lo que evidencia un crecimiento muy bajo del lomo en el rango de PC analizado (6 \%). Daza et al.

\section{BIBLIOGRAFÍA}

Aparicio, J.B. 1977. Fattening of the Iberian pig. VII. Gain in live weight with mountain pasturage diet supplemented with soya, flour control of dorsal fat deposit. Arch Zootec, 26: 97-110.

Batorek, N.; Ėandek-Potokar, M.; Bonneau, M. and Van Milgen, J. 2012. Meta-analysis of the effect of immunocastration on production performance, reproductive organs and boar taint compounds in pigs. Animal, 6: 1330-1338.

Branscheid, W.; Dobrowolski, A. and Sack, E. 1990. Simplification of the EC-reference method for the full dissection of pig carcasses. Fleischwirtsh, 70: 565-567.

Daza, A.; Olivares, A.; Cordero, G. and LópezBote, C.J. 2007. Prediction of weight of major cuts by mean slaughter or carcass weight in Iberian pigs. Span J Agric Res, 5: 318-321.

EFSA. 2004. Opinion of the Scientific Panel on Animal Health and Welfare (AHAW). doi:10.2903/ j.efsa.2004.91.

EMA. 2011. European Medicines Agency. http:// www.ema.europa.eu/ema/index.jsp?curl= pages/medicines/veterinary/medicines/000136/ vet_med_000130.jsp\&mid=WC0b01ac058001fa1c (26/9/2011).

European Declaration on alternatives to surgical castration of pigs. 2010. http://ec.europa.eu/ food/animal/welfare/farm/initiatives_en.htm (04/06/2012).
(2007), ofrecen resultados comparables a los de este trabajo en cuanto a jamones y paletas, si bien sólo trabajaron con machos Ibéricos puros castrados. No se ha encontrado otra referencia para la raza Ibérica. Se puede concluir, que la inmunocastración favorece el crecimiento de las cerdas y que la castración quirúrgica no presenta ninguna ventaja productiva, siendo de interés económico la posibilidad de predecir rendimientos con medidas sencillas e inmediatas para el productor y para el matadero.

Latorre, M.A. 2011. Factores no nutricionales que afectan a la calidad de la carne de cerdos blancos pesados. VI Congreso Mundial del Jamón. Lugo, 21-23 de septiembre.

Mateos, G.G.; Serrano, M.P.; Cámara, L.; Fuentetaja, A. y Valencia, D.G. 2009. Factores nutricionales y de manejo que influyen sobre la calidad de la canal y la carne del cerdo Ibérico en intensivo. V Congreso Mundial del Jamón, Huelva, 6 al 8 de mayo.

MAGRAMA. 2012. Ministerio de Agricultura, Alimentación y Medio Ambiente. Registro informático del lbérico (RIBER). Censos de animales y productos comercializados. http://www.magrama. gob.es/es/alimentacion/temas/calidad-agro alimentaria/calidad-comercial/mesa-del-iberico/ riber-publico/censos-animales-productos-comercializados/ (04/06/2012).

RD 1201/2005. Real Decreto de 10 de octubre, sobre protección de los animales utilizados para experimentación y otros fines científicos. (BOE nํ 252, de 21 de octubre de 2005).

RD 1469/2007. Real Decreto de 2 de noviembre, por el que se aprueba la norma de calidad para la carne, el jamón, la paleta y la caña de lomo ibéricos. (BOE nํ 264, de 3 de noviembre de 2007).

SAS Institute. 2004. User's Guide: Statistics. SAS Institute. Cary, NC. USA. 